\title{
STEM CELL RESEARCH: APPLICATIONS IN HAEMATOLOGICAL CONDITIONS
}

\author{
A.I. Anoje \\ Department of Haematology, University of Benin Teaching Hospital, Benin City
}

\section{HAEMATOPOIETIC STEM CELL TRANS-PLANTATION (HSCT)}

\section{DEFINITION}

Haematopoietic Stem Cell Trans-plantation is a medical procedure in the field of haematology and oncology that involves transplantation of haematopoietic stem cells (HSC). It is most often performed for people with diseases of the blood or bone narrow or certain types of cancers.

\section{PRINCIPLES}

Most recipients of HSCT are leukaemia patients or others who would benefit from treatment with high doses of chemotherapy or total body irradiation (TBI). Other beneficiaries of HSC include Paediatric cases that require bone marrow transplant; cases in which the patient has an inborn defect such as severe combined immunodeficiency or congenital neutropenia and was born with defective stem cells. Children or adult with aplastic anaemia have lost their stem cells after birth and may not require such high doses of chemotherapy and irradiation prior to transplant. In this case there is greater need for immunosuppressive agents. Other conditions that bone marrow transplants are considered include thalassemia major, sickle cell disease, Myelodysplastic syndrome, lymphoma and multiple myeloma. More recently nonmyeloablative, or so called "mini transplant" procedures have been developed which do not require such large doses of chemotherapy and radiation. This has allowed HSCT to be conducted in older patients without the need for hospitalization.

\section{STEM CELL COLLECTION}

\section{Types of Donors}

There are two major types of bone marrow transplantation namely; Autologous and Allogenic transplantations.

Autologous: Bone marrow transplantation involves isolation of HSC from a patient suffering from a blood cancer usually; freezing of the isolated stem cells; storage of the frozen stem cells; medical treatment of the patient that usually results in destruction of the remaining stem cells in the body; and then return of the stored stem cells back to the body. Autologous transplants have the advantage of a lower risk of graft rejection and Graft Versus Host Disease.

Allogeneic: Allogenic bone marrow transplantation involves two people, one is the (normal) donor and one is the (patient) recipient. Allogeneic HSC donors must have a tissue (HLA) type that matches the recipient and in addition, the recipient requires immuno suppressive medication. Allogeneic 
transplant donors may be related (usually a sibling) or unrelated volunteers.

\section{Donor selection}

A major limitation of allogeneic bone marrow transplantation is a shortage of donors. To avoid rejection of the transplanted stem cells or severe Graft Vs Host Disease (GVHD), the donor should have the same human leucocyte antigens (HLA) as the recipient. About 25 to 30 percent of potential HSCT recipient have an HLA - identical sibling. For other recipients, registries whilst available, of volunteer unrelated donors can be quickly searched in order to find a potential HLA match. If an exact match cannot be found, a partially matched donor can be used. However, the use of mismatched donors may increase the risk of graft rejection or severe graft - versus host disease.

A compatible donor is found by doing additional HLA - testing from the blood of potential donors. The HLA genes fall into two categories (type I and type II). In general, mismatches of the type I genes (i.e. HLA - A, HLA - B or HLA - C) increase the risk of graft rejection. $A$ mismatch of an HLA type II gene (i.e. HLA - DR, HLA - ADOBI) increases the risk of GVHD. In addition a genetic mismatch as small as a single DNA base pair is significant. So perfect matches require knowledge of the exact DNA sequence of these genes for both donor and recipient. Leading transplant centres currently perform testing for all five of these HLA genes before declaring that a donor and recipient are HLA identical.

\section{Sources of HSC}

In the case of bone marrow transplant, the HSC are moved from a large bone of the donor, typically the pelvis, through a large needle that reaches the centre of the bone. The technique is referred to as a bone marrow harvest and is performed under general anaesthesia because hundreds of insertions of the needle may be required to obtained sufficient material.

Peripheral blood stem cells - are now the most common source of stem cells for HSCT. They are collected from the blood through a process known as apheresis - the donor's blood is withdrawn through a sterile needle in one arm and passed through a machine that removes white blood cells, the red blood cells are returned to the donor. The peripheral stem cell yield is boosted with daily subcutaneous injections of filgrastim.

Umbilical cord blood - is obtained when a mother donates her infant's umbilical cord and placenta after birth. Cord blood has a higher concentration of HSC than is normally found in adult blood. However, the small quantity of blood obtained from the umbilical cord (typically about $50 \mathrm{ml}$ ) makes it more suitable for transplantation into small children than in adults. Newer techniques using ex-vivo expansion of cord blood units or the use of two cord blood units from different donors are being explored to allow cord blood transplant to be used in adults.

\section{Storage of HSC}

Unlike other organs, bone marrow cells can be frozen for prolonged time periods (cryopreserved/ without damaging too many cells). This is necessary for autologuous HSC because the cells must be 
harvested months in advance of the transplant treatment. In the case of allogeneic transplants, HSC are preferred in order to avoid cell loss that might occur during the freezing and, thawing process. Allogeneic cord blood is stored frozen at a cord blood bank because it is only obtainable at the time of childbirth. To cryopreserve HSC, a preservative DMSO must be added and the cells must be cooled very slowly in a control rate freezer to prevent osmotic cellular injury during ice crystal formation. HSC may be stored for years in a cryofreezer which typically utilizes liquid nitrogen because it is non-toxic and it is very cold (boiling point $-196^{\circ} \mathrm{C}$ ).

\section{Conditioning Regimens}

Ablation of the diseased immune system is the goal of the conditioning regimen. It is a preparative regimen with the use of chemotherapy or irradiation. The purpose is to help eradicate the patient's diseases prior to the infusion of HSC and to suppress immune reactions. Chemotherapy drugs and radiation both damage DNA in the cell nucleus which kills rapidly dividing cells by triggering a self-destructive mechanism called apoptosis. Bone marrow cells divide frequently and are particularly sensitive in this treatment. The bone marrow can be ablated at doses that cause minimal injury to other tissues. In allogeneic transplant, a combination of cyclophosphamide with busulfan or total body irradiation is commonly employed. This treatment also has immunosuppressive effects which prevents rejection of the HSC by the recipient immune system. Autologous transplants may also use this conditioning regimens.
Non-Myeloablative allogeneic HSCT is a newer treatment approach which uses lower doses of chemotherapy and irradiation which are too low to eradicate all of the bone marrow cells of a recipient. Instead non-myeloablative transplant rely on the graft versus tumour effect for their benefit. They do require high doses of immunosuppressive agents in the early stage of treatment. This leads to a state of mixed chimerism early after transplant where both recipient and donor HSC coexist in the bone marrow space, decreasing doses of immunosuppressive therapy than allow donor $T$ - cells to eradicate the remaining recipient $\mathrm{HSC}$ and to induce GVHD and the graft versus tumour effect.

Non - myeloablastive (or "min") allogeneic transplant, because of their gentler conditioning regimens, are associated with a lower risk of transplant - related mortality and therefore allow patient who are considered too high risk for conventional allogeneic HSCT (because of age or other comorbidities) to undergo potentially curative therapy for their disease.

\section{TRANSPLANTATION AND ENGRAFTMENT}

HSC are infused into the blood stream of the recipient through an intravenous (i.v) catheter. Like any other i.v. fluid the HSC briefly circulate in the blood stream and then home into the bone marrow spaces where they grow and start to produce blood cells. After several weeks of growth in the bone marrow, expansion of HSC and their progeny is sufficient to normalize the blood cell counts and alleviate the need for red blood cells and platelets transfusion. 
Side effects and complications

Bone marrow transplantation is associated with a fairly high mortality ( $10 \%$ or higher), which limits its use to conditions that are themselves essentially life threatening. Major causes of complications are sepsis, graft-versus host disease and venoocclusive disease.

\section{Infection}

Bone marrow transplantation usually requires that the recipients own bone marrow is destroyed (myeloablation). Prior to "engraftment", patients may go for several weeks without appreciable numbers of white blood cells to help fight infection. This puts a patient at risk of infections, sepsis and septic shock despite prophylactic antibiotics. The immunosuppressive agent employed in allogeneic transplant for the prevention or treatment of GVHD further increase the risk of opportunistic infection. Immunosuppressive drugs are given for a period of 6 months after a transplantation or much longer if required for the treatment of GVHD. Transplant patients lose their acquired immunity, for example immunity to childhood diseases such as measles or polio. For this reasons transplant patient must be revaccinated with childhood vaccines once they are off immuno-suppressive medications.

\section{GRAFT VERSUS HOST DISEASE}

GVHD is an inflammatory disease that is unique to allogeneic transplantation. It is an attack of the "new" bone marrow's immune cells against the recipient's tissues. This can occur even if the donor and recipient are HLA - identical because the immune system can still recognise other differences between their tissues. It is aptly named graft versus host disease because bone marrow transplantation is the only transplant procedure in which trans-planted cells must accept the body rather than the body accepting the new cells. Acute GVHD typically occurs in the first 3 months after transplantation and may involve the skin, intestine or the liver. Corticosteroids such as prednisolone are a standard treatment. Chronic GVHD may also develop after allogeneic transplant and is the major source of late complications. In addition to inflammation, chronic GVHD may lead to the development of fibrosis or scar tissue, similar to scleroderma or other autoimmune disease and may cause functional disability and the need for prolonged immunosuppressive therapy.

\section{Graft versus tumour effect}

The beneficial aspect of Graft versus Host is known as "graft versus tumour" or "graft versus leukaemia". For example leukaemia patient with chronic GVHD after an allogeneic transplant have lower risk of leukaemia relapse. This is due to therapeutic immune reaction of the grafted donor lymphocytes against the disease bone marrow of the recipient. This lower rate of relapse accounts for the increased success rate of allogeneic transplant compared to transplant from identical twins, and indicates that allogeneic HSCT is a form of immunotherapy. Graft vs tumour is also the major mechanism of benefit of non-myeloablative transplant which do not employ high dose chemotherapy or radiation.

\section{CONDITIONS TREATED WITH BONE MARROW TRANSPLANT Acquired}

1. Acute lymphocytic leukaemia 
2. Acute myelogenous leukaemia

3. Aplastic anaemia

4. Chronic myelogenous leukaemia - accelerated phase or blast crises

5. Hodgkin's disease

6. Multiple myeloma

7. Myelodysplasia

8. Non - Hodgkin's lymphoma

9. Paroxysmal nocturnal haemoglobinuria (PNH) - severe aplasia

10. Radiation poisoning.

\section{Congenital}

1. Adrenoleukodystrophy

2. Hurler syndrome

3. Kostmann syndrome

4. Drabbe disease

5. Metachromatic leukodystrophy

6. Hemophagocytic lymphohistocytosis (HLH)

7. Wiskot - Aldrich syndrome

8. Some inborn errors of metabolism.

\section{SUMMARY AND CONCLUSION}

Transplantation of allogeneic haema-topoietic stem cells is an established treatment for some relatively rare malignant blood diseases, congenital or acquired diseases with severe bone marrow failure, and certain metabolic diseases. The best documentation is found for treatment using bone marrow or stem cells mobilized to peripheral blood from HLA - compatible donors both related and unrelated. Since 1990, cord blood from related or unrelated donors has been used as an alternative to stem cells from bone marrow or blood.

\section{REFERENCES}

1. Thomas ED, Lochte HL, Lu WC et al. Intravenous infusion of bone marrow in patients receiving radiation and chemotherapy. New Engl Med. J. 1957; 157: 491 - 496.

2. Socie A, Gerarol L. Busulfan plus cyclophosphomide compared with total - body irradiation plus cyclosphosphomide before marrow transplantation for myeloid leukaemia: long term follow up of 4 randomized studies: Blood. 2001; 98(13): 3569 - 3574.

3. Richarolson PG. Multi-institutional use of difibrotide in 88 patients after stem cell transplantation with severe veno-occlusive disease and multisystem organ failure response without significant toxicity in a high-risk population and factors predictive of outcome. Blood. 2002; 100(13): $4337-43$.

4. Crugielmi PT. Autologous bone marrow transplantation as compared with salvage chemotherapy in relapses of chemotherapy - sensitive nonHodgkin's lymphoma. New England Journal of medicine. 1995; 333(23): 1540 - 5.

5. Barker JN. Transplantation of 2 partially HLA - method umbilical cord blood units to enhance engraftment in adult with haemotologic malignancy. Blood 2005; 105(3): $134-1347$. 